\begin{tabular}{|l|c|c|c|c|}
\hline Cuadernos I. Geográfica & 24 & pp. 25-38 & Logroño & 1998 \\
\hline
\end{tabular}

\title{
COMPORTAMIENTO DE LA HUMEDAD DEL SUELO EN UNA PEQUEÑA CUENCA HIDROGRÁFICA DE LA DEHESA EXTREMEÑ̃ (GUADALPERALÓN, CÁCERES).
}

\section{A. CEBALLOS BARBANCHO (1) \\ S. SCHNABEL (1)}

RESUMEN: La bumedad del suelo depende de sus propiedades físicas y se muestra como un factor determinante por la influencia que ejerce sobre los procesos de infiltración y escorrentía. El principal objetivo del presente trabajo es el estudio de la bumedad del suelo en una pequeña cuenca bidrográfica característica de la debesa extremeña en relación con el espacio poroso. Los resultados muestran una distribución espacial de la bumedad edáfica similar a la densidad y porosidad. La correlación entre porosidad y humedad es alta. No obstante, la existencia de costras superficiales y concavidades rellenas de sedimentos alteran esta relación.

ABSTRACT: Soil moisture depends on the soil physical characteristics, being a determinant factor in infiltration and runoff processes. The principal aim of this paper is the analysis of soil moisture in relation with porosity in a small drainage basin representative of the Extremenian debesa. The results show a similar spatial distribution of soil moisture, density and porosity. The correlation between porosity and moisture is bigh. Nevertheless, the existence of surface crusts and concavities filled with sediments disturb this relation.

Palabras clave: humedad del suelo, capacidad de campo, porosidad, densidad, dehesa.

Key words: soil moisture, field capacity, porosity, density, dehesa.

(1) Área de Geografia Física. Dpto. de Geografia y O.T. Facultad de Filosofía y Letras. Avda. de la Universidad, s/n. 10071 - Cáceres. 
A. CEBALLOS BARBANCHO - S. SCHNABEL

\section{Introducción}

Desde 1990 el Área de Geografía Física de la Universidad de Extremadura está estudiando los procesos hidrológicos y sedimentológicos en una cuenca hidrográfica experimental representativa del ecosistema dehesa (Gómez Amelia y Schnabel, 1992). La dehesa supone más de la mitad de la superficie agraria útil de las provincias del suroeste español y todavía son escasos los estudios sobre los procesos físicos que operan en la misma.

En el establecimiento del balance de agua de la cuenca no se ha encontrado una relación estadística entre humedad del suelo y escorrentía superficial (Ceballos, 1997), sin embargo se ha observado que cuando los suelos alcanzan su capacidad de campo, y se aproximan a su estado de saturación, los coeficientes de escorrentía son elevados. El conocimiento de las propiedades físicas del suelo es esencial en el desarrollo de las investigaciones geomorfológicas e hidrológicas por el control que ejercen sobre los procesos de infiltración y escorrentía superficial (Romero Díaz, 1986; Martínez Fernández, 1994; Martínez Fernández y López Bermúdez, 1996). Entre las mismas destaca la porosidad que podemos definir como la porción de volumen de suelo ocupada por aire y agua, y comprende tanto la porosidad no capilar o macroporosidad y la porosidad capilar o microporosidad, siendo la suma de ambas la porosidad total (Guitián y Carballas, 1976). El volumen de espacio poroso y la distribución del tamaño de los poros son los rasgos fundamentales que explican la capacidad de retención hídrica de un suelo (Courtney y Trudgill, 1984), ya que existe una relación causa-efecto entre la organización del espacio poroso y los valores de la humedad del suelo (Ingelmo y Cuadrado,1986). El volumen de suelo ocupado por materia sólida (densidad) dificulta la circulación de agua a través del él, lo que se traduce en menores tasas de infiltración (Cerdà, 1995).

El objetivo del presente trabajo es el estudio del comportamiento de la humedad edáfica de la cuenca, prestando una especial atención a su relación con la distribución del espacio poroso.

\section{Zona de estudio}

La cuenca de Guadalperalón, con una superficie de 35,4 hectáreas, se localiza $22 \mathrm{Km}$ al NE de la ciudad de Cáceres, dentro de la cuenca del río Magasca, perteneciente a la red hidrográfica del Tajo (figura 1). 

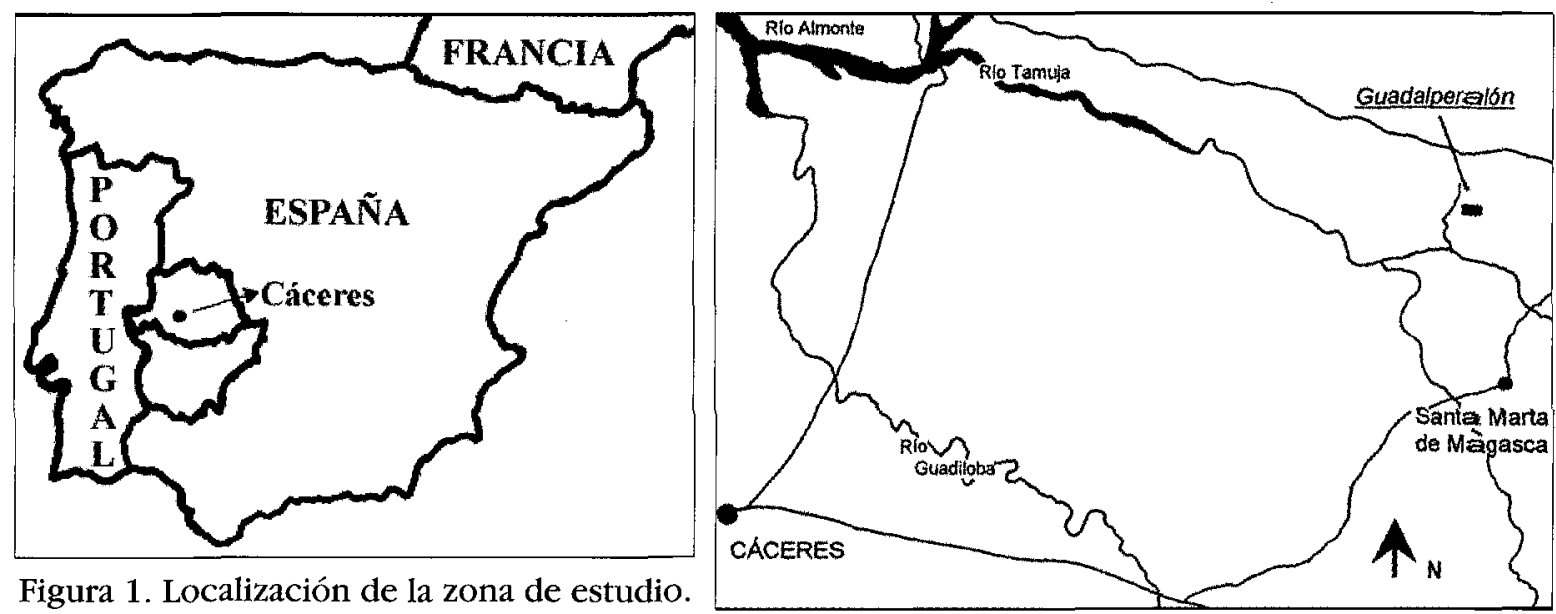

Figura 1. Localización de la zona de estudio.

Se halla integrada en la mitad oriental de la superficie de erosión finimiocena que conforma la Penillanura Cacereña (Gómez Amelia, 1985). Los suelos se han desarrollado sobre un sustrato pizarroso (Complejo Esquisto Grauváquico) y se encuadran en el grupo de los Leptosoles y Regosoles según sus características topográficas y/o fisiográficas (Driessen y Dudal, 1991). La profundidad de los mismos oscila entre los 0 y $120 \mathrm{~cm}$, su textura es principalmente franca, la estructura granular y los valores de materia orgánica bajos (2,7\%).

El clima es meditarráneo, con una temperatura media anual de $16^{\circ} \mathrm{C}$ y una precipitación anual media de $512 \mathrm{~mm}$ distribuidos en 85 días de lluvia. Destaca la alta variabilidad, tanto anual como interanual, de las precipitaciones, predominando el número de años con valores inferiores a los medios (Ceballos, 1997).

La vegetación potencial pertenece a la serie climatófila mesomediterránea luso-extremadurense de la encina (Quercus rotundifolia) (Ladero Álvarez, 1987). El aprovechamiento principal de la dehesa de Guadalperalón es el ganadero, con una cabaña constituida esencialmente por oveja merina y unas cargas que oscilan entre 0 y 2,3 cabezas/ $\mathrm{Ha}$.

Estudios anteriores (Schnabel, 1995 y 1997) han establecido una división espacial de la cuenca en 5 unidades atendiendo a la distribución y evolución del grado de cobertura vegetal de la misma, así como a la composición del pastizal (tabla 1). 
A. CEBALLOS BARBANCHO - S. SCHNABEL

Tabla 1. Descripción de las unidades espaciales de la cuenca según la división propuesta por SCHNABEL (1995 y 1997).

\begin{tabular}{|cll|}
\hline \multirow{2}{*}{ Unidad } & \multicolumn{1}{c|}{ Descripción } & \% de superficie \\
\hline \multirow{2}{*}{ G1 } & $\begin{array}{l}\text { Vertientes arboladas con densidad variable } \\
\text { y estrato herbáceo denso. }\end{array}$ & 7,8 \\
\hline G2 & $\begin{array}{l}\text { Vertientes arboladas con densidad variable } \\
\text { y cubierta herbácea media. }\end{array}$ & 50,9 \\
\hline G3 & $\begin{array}{l}\text { Áreas rocosas, con litosuelos, sustrato arbustivo } \\
\text { de cantueso (Lavandula stoecbas) y sin arbolado. }\end{array}$ & 29,8 \\
\hline AC & $\begin{array}{l}\text { Zona de acumulación (fondos de valle) con } \\
\text { suelos profundos y estrato herbáceo denso. }\end{array}$ & 11,5 \\
\hline TR & $\begin{array}{l}\text { Zona de las vertientes arboladas (G1 y G2) bajo } \\
\text { la influencia directa de la copa de los árboles. }\end{array}$ & \\
\hline
\end{tabular}

\section{Metodología}

La determinación de la densidad y porosidad del nivel superficial del suelo se ha obtenido analizando una serie de muestras recogidas en el campo con un anillo de $5 \mathrm{~cm}$ de altura y un volumen de $98,12 \mathrm{~cm}^{3}$. Tras secar las muestras de suelo en la estufa a una temperatura de $105^{\circ} \mathrm{C}$ durante 24 horas, la densidad aparente se obtuvo dividiendo el valor del peso de la muestra, ya seca, por el volumen del cilindro utilizado $\left(98,12 \mathrm{gr} \mathrm{cm}^{-3}\right)$. Para el cálculo del espacio poroso se ha aplicado la siguiente fórmula:

$$
\% \text { espacio poroso }=100-(\mathrm{Db} / \mathrm{Dp} \times 100)
$$

donde

$\mathrm{Db}=$ "Bulk density" o densidad aparente que define la masa $\left(\mathrm{gr} \mathrm{cm}^{-3}\right)$ de una unidad de volumen de suelo seco. Este volumen incluye tanto los cuerpos sólidos como las porosidades.

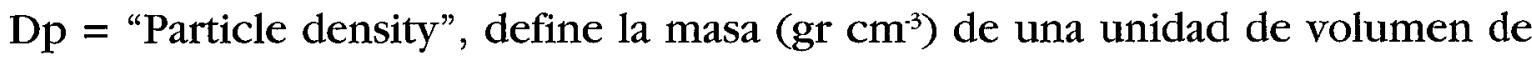
suelo exclusivamente sólido. Para cálculos generales se utiliza un valor promedio equivalente $2,6 \mathrm{gr} \mathrm{cm}^{-3}$ (Duchafour, 1987), salvo que los suelos analizados posean una alta proporción de materia orgánica, circunstancia que no se da en los suelos del espacio estudiado.

El contenido de humedad se ha obtenido mediante la aplicación del método gravimétrico (Reynolds, 1970) que se basa en el cálculo de la humedad por 
diferencia de peso del suelo antes y después de secarse en una estufa a $105{ }^{\circ} \mathrm{C}$ durante 24 horas:

$$
\% \text { humedad del suelo }=(\mathrm{A}-\mathrm{B} / \mathrm{B}) \times 100
$$

donde

$\mathrm{A}=$ peso de la muestra húmeda

$B=$ peso de la muestra seca.

Se utilizaron las mismas muestras para la determinación de la densidad, porosidad y humedad del suelo. Las muestras se tomaron en intervalos irregulares de tiempo según los sucesos de precipitación. Las principales ventajas del método gravimétrico son su facil manejo y cálculo, y escaso costo; siendo su principal inconveniente la alteración y destrucción del terreno estudiado (Martínez Fernández y López Bermúdez, 1996).

La capacidad de campo se determinó sobre el propio terreno, tratándose de una medida que engloba el agua retenida en el suelo más una fracción del agua de gravitación de flujo lento (Duchafour, 1987).

El período de muestreo utilizado para la realización del presente trabajo ha sido el año hidrológico 1995-96. En dicho año se registró una precipitación de $720 \mathrm{~mm}$ irregularmente distribuidos a lo largo del mismo como puede apreciarse en la figura 2 (la estación más lluviosa es el invierno con un 57,7\% del total, mientras que en los meses veraniegos no se registró ninguna precipitación).

\section{Resultados y discusión}

Tras el análisis de 550 mediciones en diferentes puntos representativos de la variabilidad espacial de la cuenca se ha obtenido un valor medio de porosidad de 51,5 \% y 1,26 gr cm $\mathrm{cm}^{3}$ de densidad. Considerando la distribución espacial de la porosidad del horizonte superficial de los suelos de la cuenca puede comprobarse como existe una relación entre la misma y las diversas unidades espaciales establecidas para el estudio de la vegetación (ANOVA: F=16.594; $\mathrm{df}=4,13 ; \mathrm{p}<0.00005$ ).

Tabla 2. Porosidad y capacidad de campo media de las diferentes unidades espaciales de la cuenca de Guadalperalón.

\begin{tabular}{|lccccc|}
\hline \multicolumn{1}{|c}{ Unidad } & G1 & G2 & G3 & AC & TR \\
\hline Porosidad (\%) & 51,9 & 47,8 & 47,5 & 48,2 & 63,0 \\
Capacidad de campo (\%) & 29,7 & 26,2 & 21,7 & 27,5 & 39,2 \\
\hline
\end{tabular}


Los suelos más densos y menos porosos se corresponden con las tres unidades que presentan un mayor nivel de degradación (G3, G2 y AC): los suelos menos porosos corresponden a G3, que es la unidad que presenta suelos más deteriorados. La unidad G2 también se caracteriza por unos suelos densos y poco porosos que se relacionan con sus altos valores anuales de suelo desnudo. Finalmente, dentro de este primer grupo, la unidad AC sería la que presenta unos valores menores de densidad y mayores de porosidad, circunstancias relacionadas con una mayor capacidad de campo, la ausencia de porcentajes significativos de suelo desnudo durante el año y la permanencia continuada de pastos.

La unidad G1, en relación con el bloque anterior, presenta unos valores bajos de densidad y altos de porosidad. Esta circunstancia puede relacionarse con la abundancia de pastos en esta zona durante gran parte del año debido a la práctica del redileo (concentración del ganado en un aprisco para que con sus excrementos abone el suelo) a comienzos del verano de 1991 (Gómez Amelia y Schnabel, 1996), así como a su exposición (umbría).

Finalmente, la unidad con valores sensiblemente superiores de porosidad e inferiores de densidad es la comprendida por aquellos puntos situados bajo el efecto de las copas de las encinas (TR). Este hecho se explica fundamentalmente por la presencia de abundante materia orgánica en el horizonte superficial del suelo.

La evolución de la humedad del suelo durante el año depende del total de precipitación caída y su reparto anual. Zhang y Berndtsson (1988) señalan que en los horizontes superficiales del suelo, la precipitación y la vegetación son los factores principales que influyen en la variabilidad de la humedad del suelo. La figura 2 muestra la evolución temporal de las precipitaciones diarias en la cuenca de Guadalperalón en el año 1995-96 y de la humedad del horizonte superficial de los suelos de la cuenca, lo cual ha permitido delimitar un umbral medio de capacidad de campo en torno a un $25 \%$. La cuenca alcanza su capacidad de campo media a finales del mes de diciembre, debido a las abundantes precipitaciones previas $(150 \mathrm{~mm})$. En ese estado permanece hasta mediados del mes de febrero. Debido a las lluvias caídas durante la primavera, de nuevo alcanza la capacidad de campo en marzo y en mayo. Entre mediados del mes de mayo y el final del año hidrológico no se registra ninguna precipitación, lo cual se traduce en una caída en picado de la curva de humedad hasta alcanzar un valor permanente en torno al 1,3\% (reserva fija o agua higroscópica). 
COMPORTAMIENTO DE LA HUMEDAD DEL SUELO EN UNA PEQUEÑA CUENCA HIDROGRÁFICA

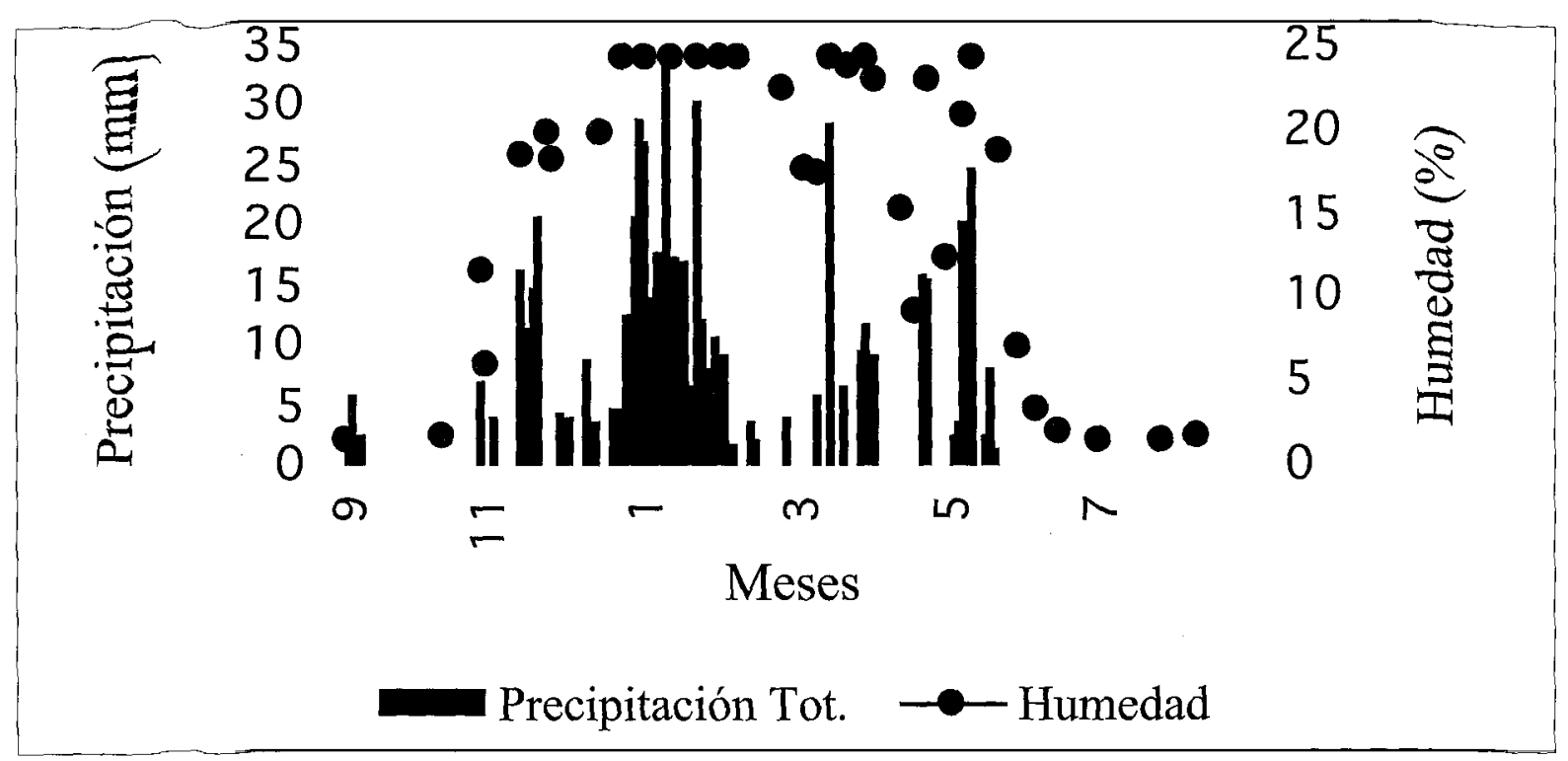

Figura 2. Evolución temporal de la humedad media de los suelos de la cuenca de Guadalperalón en relacióncon la precipitación diaria. Año 1995-96.

Duniny Aston (1981) demuestran que es factible delimitar diferentes zonas hidrológicas en una cuenca siguiendo como criterio la uniformidad del comportamiento de la humedad del suelo, a pesar de la apreciable variabilidad de la misma y de la conductividad hidraúlica en pocos metros de distancia (Price y Bauer, 1984). En la cuenca de Guadalperalón se ha apreciado un distinto comportamiento de la humedad que ha permitido agrupar los puntos de muestreo en diversas unidades que coinciden plenamente con las ya establecidas para el estudio de la vegetación y parámetros físicos del suelo (densidad y porosidad).

La unidad con suelos más deteriorados, altos valores de densidad y bajos de porosidad, encostramiento de los milímetros superficiales y exposición en solana (unidad G3), es aquella que tiene la menor capacidad de retención hídrica. En el lado opuesto, la unidad con mayores valores de humedad corresponde a las áreas situadas bajo la proyección de las copas de las encinas (unidad TR), como consecuencia de la alta porosidad del horizonte superficial del suelo debido a la presencia de materia orgánica (descomposición de la biomasa procedente del propio árbol).

La unidad G1, con una superficie abundante en pastos, porosidad alta y exposición en umbría, es la unidad que registra el segundo valor más alto de retención hídrica. Los pies de ladera (unidad AC), con suelos más profundos, alcanzan la capacidad de campo con un $27,5 \%$ de humedad, no obstante se han medido valores sensiblemente superiores a esta cifra debido a la articulación de flujo subsuperficial durante y después de períodos de precipitaciones 
A. CEBALLOS BARBANCHO - $S$. SCHNABEL

continuadas (Harr, 1977). Finalmente, la unidad G2 tiene un máximo de humedad del $26,2 \% \mathrm{y}$, debido principalmente a la poca profundidad de los suelos, muestra una mayor velocidad de secado (evaporación) respecto a otras unidades.

Tabla 3. Serie de mediciones. Coeficiente de correlación $\left(r^{2}\right)$ entre porosidad y bumedad, valor-P, Hum.Med.: bumedad del suelo media de la serie, Hum.Es: desviación estándar de la serie de bumedad, Hum.C.V.: coeficiente de variación de la serie de bumedad, Por.Med.: porosidad media de la serie, Por.Es: desviación estándar de la serie de porosidad, Por.C.V.: coeficiente de variación de la serie de porosidad.

\begin{tabular}{rrllrrrrrr}
\hline $\begin{array}{r}\text { N. } \\
\text { muestra }\end{array}$ & Fecha & $r^{2}$ & valor-p & $\begin{array}{c}\text { Hum. } \\
\text { Med (\%) }\end{array}$ & \multicolumn{1}{c}{$\begin{array}{r}\text { Hum. } \\
\text { Es. }\end{array}$} & \multicolumn{1}{c}{ Hum. } & $\begin{array}{c}\text { Por.Med } \\
(\%)\end{array}$ & Por.Es & Por.C.V. \\
\hline 1 & $09-10-95$ & 0,78 & 0,000 & 1,70 & 0,80 & 46,74 & 47,26 & 7,48 & 15,83 \\
2 & $11-11-95$ & 0,73 & 0,000 & 19,15 & 5,90 & 30,81 & 50,14 & 7,57 & 15,09 \\
3 & $21-11-95$ & 0,42 & 0,003 & 19,84 & 4,22 & 21,27 & 50,43 & 5,88 & 11,67 \\
4 & $24-11-95$ & 0,58 & 0,000 & 18,55 & 5,47 & 29,48 & 50,54 & 7,78 & 15,39 \\
5 & $13-12-95$ & 0,61 & 0,000 & 20,04 & 4,92 & 24,54 & 50,72 & 7,85 & 15,48 \\
6 & $21-12-95$ & 0,90 & 0,000 & 25,26 & 5,30 & 20,99 & 51,45 & 8,87 & 17,23 \\
7 & $07-02-96$ & 0,68 & 0,000 & 29,30 & 9,64 & 32,88 & 50,50 & 6,65 & 13,17 \\
8 & $26-02-96$ & 0,59 & 0,000 & 24,30 & 10,79 & 44,41 & 50,70 & 9,63 & 18,99 \\
9 & $04-03-96$ & 0,35 & 0,007 & 20,30 & 10,24 & 50,46 & 51,30 & 8,24 & 16,06 \\
10 & $09-03-96$ & 0,34 & 0,008 & 19,60 & 8,18 & 41,74 & 50,10 & 7,71 & 15,39 \\
11 & $15-03-96$ & 0,78 & 0,000 & 27,38 & 10,75 & 39,25 & 51,84 & 7,71 & 14,87 \\
12 & $21-03-96$ & 0,43 & 0,002 & 24,64 & 7,01 & 28,44 & 52,40 & 8,04 & 15,33 \\
13 & $28-03-96$ & 0,80 & 0,000 & 26,77 & 9,82 & 36,68 & 52,32 & 7,82 & 14,94 \\
14 & $03-04-96$ & 0,34 & 0,009 & 23,44 & 6,39 & 27,26 & 50,09 & 5,62 & 11,22 \\
15 & $12-04-96$ & 0,24 & 0,035 & 17,69 & 10,02 & 56,64 & 52,17 & 9,69 & 18,57 \\
16 & $19-04-96$ & 0,36 & 0,006 & 11,97 & 10,32 & 86,22 & 52,92 & 8,43 & 15,92 \\
17 & $23-04-96$ & 0,77 & 0,000 & 25,48 & 10,02 & 39,33 & 55,20 & 7,08 & 12,83 \\
18 & $01-05-96$ & 0,37 & 0,006 & 14,44 & 9,01 & 62,37 & 54,86 & 8,08 & 14,73 \\
19 & $07-05-96$ & 0,52 & 0,000 & 22,52 & 6,77 & 30,06 & 51,53 & 7,28 & 14,13 \\
20 & $11-05-96$ & 0,78 & 0,000 & 26,88 & 10,75 & 40,00 & 51,96 & 8,81 & 16,96 \\
21 & $21-05-96$ & 0,68 & 0,000 & 21,13 & 10,52 & 49,78 & 52,98 & 7,30 & 13,78 \\
22 & $28-05-96$ & 0,39 & 0,004 & 9,87 & 9,76 & 98,89 & 55,01 & 7,72 & 14,03 \\
23 & $06-06-96$ & 0,17 & 0,082 & 4,29 & 4,04 & 94,27 & 54,06 & 6,87 & 12,70 \\
24 & $14-06-96$ & 0,24 & 0,033 & 2,90 & 3,10 & 106,79 & 53,97 & 6,24 & 11,56 \\
25 & $09-08-96$ & 0,53 & 0,000 & 1,83 & 1,31 & 71,48 & 56,56 & 8,88 & 15,70
\end{tabular}


Existe un grado alto de relación entre la porosidad y la capacidad de retención hídrica del suelo (tabla 3). Los mayores coeficientes de correlación $\left(\mathbf{r}^{2}>\right.$ 0,75 ) se han registrado en situaciones de total uniformidad del estado de los suelos de la cuenca: bien en situación de total sequía, donde el espacio poroso sólo contenía la reserva fija o el agua higroscópica (muestra $\mathrm{n} .^{\circ}$ 1), o bien con valores de humedad muy altos (muestras n. ${ }^{\circ}$ 6, 11, 13, 17 y 20) (figura 3). En el resto de los casos, el coeficiente de correlación decrece, no por una dispersión de la nube de puntos respecto a la tendencia marcada por la recta de regresión, sino por la existencia de residuos ("outliers") muy localizados.

En el caso de la muestra n..$^{\circ} 14$, los dos puntos contenidos en la elipse corresponden a la unidad G3, donde es frecuente la existencia de costras milimétricas superficiales que dificultan los procesos de infiltración y explican el bajo valor de la humedad del suelo. Diversos trabajos (Imeson, 1984; Navas, 1988) afirman que la presencia de costras superficiales es uno de los factores con mayor influencia en el proceso de infiltración. La situación que refleja el gráfico de la serie $n^{\circ} 24$ (figura 3) es mucho más frecuente en la cuenca. Los dos puntos incluidos en la elipse pertenecen a la zona de acumulación, donde son característicos los mecanismos de reinfiltración del agua procedente de las vertientes y se registra flujo subsuperficial; lo que explica que los valores de humedad edáfica, a lo largo del tiempo, sean mucho mayores que el resto de las unidades, donde la velocidad de secado del espacio poroso es superior. Este hecho indica una cierta influencia de la posición topográfica sobre la humedad del suelo. Aunque no se ha realizado un estudio exhaustivo de las características texturales de los suelos de la cuenca, los suelos menos arenosos y más limosos se han localizado en los fondos de valle, lo cual incide en un peor drenaje interno y menores pérdidas por evaporación. El mismo comportamiento se ha observado en otras áreas investigadas (Cerdà, 1996).

Si en el caso de la humedad del suelo se aprecia una importante variabilidad temporal dependiendo principalmente del ritmo pluviométrico, en el caso de la porosidad hay una cierta estabilidad de los valores promedios a lo largo del año de medición, como se puede observar en la escasa variabilidad del coeficiente de variación (tabla 3).

En algunas zonas muy puntuales de la cuenca, como son las veredas utilizadas por el ganado (ovejas merinas) en sus desplazamientos, se ha observado un incremento notable de la densidad del suelo $\left(1,43 \mathrm{gr} \mathrm{cm}^{-3}\right)$ y un decrecimiento de la porosidad (44,9\%). Diversos estudios han demostrado como el pisoteo del ganado compacta y encostra los suelos, con una reducción significativa de la capacidad de retención hídrica y de la tasa de infiltración (Gamougoun et al., 1984; Mulholland y Fullen, 1991). 
A. CEBALLOS BARBANCHO - S. SCHNABEL
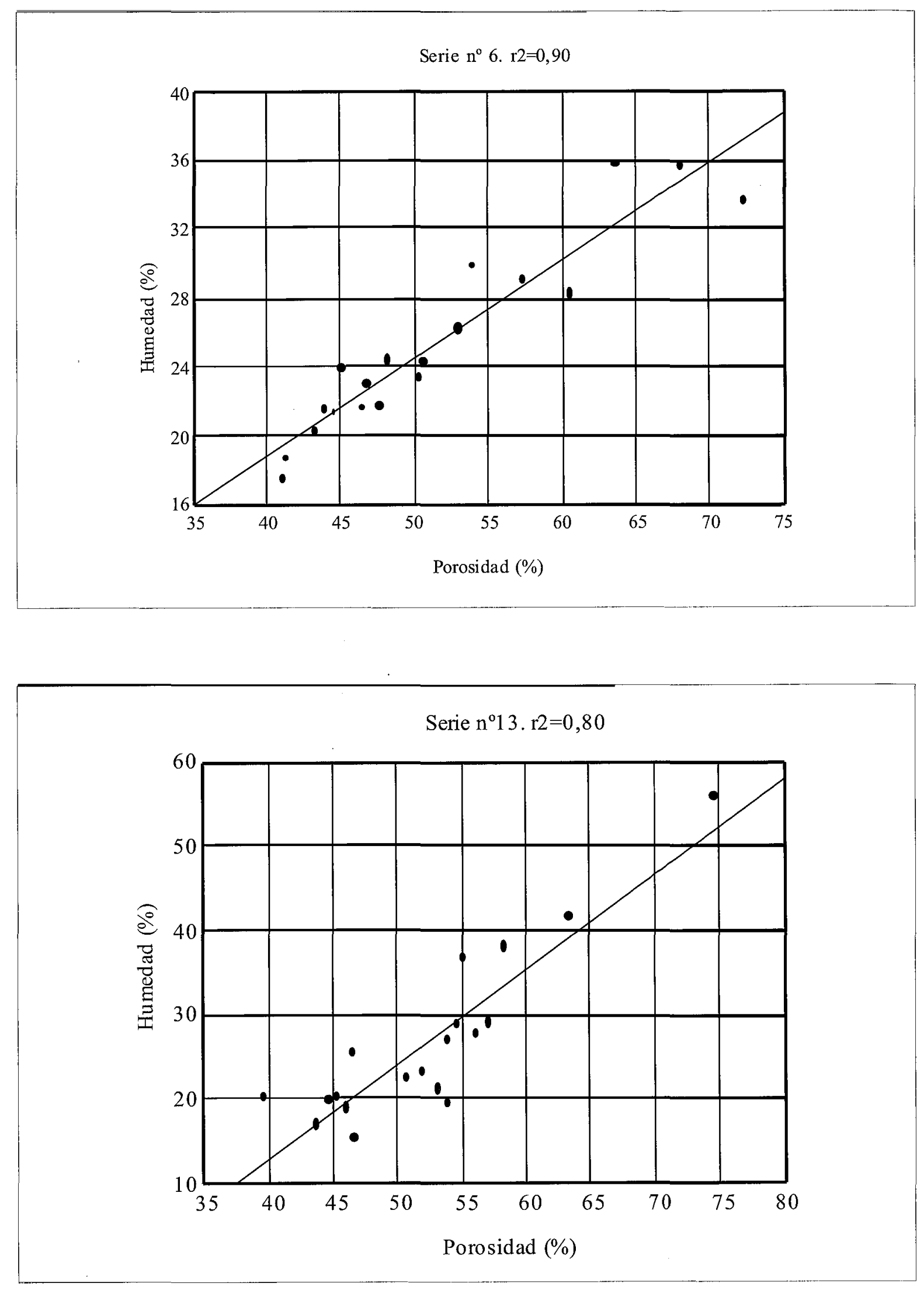

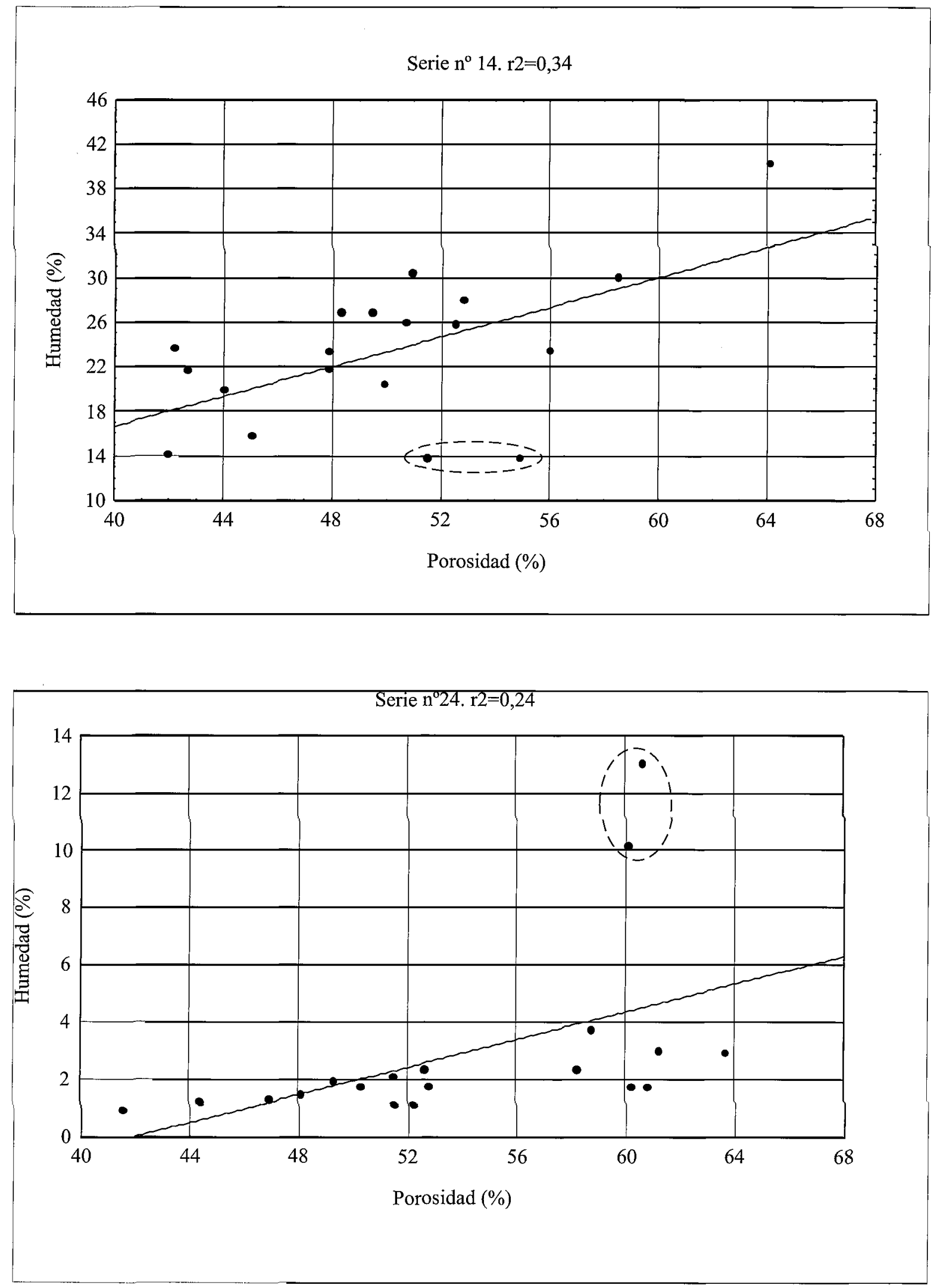

Figura 3. Correlaciones lineales entre porosidad y humedad del suelo. Series n ${ }^{\circ} 6,13,14$ y 24 . 
A. CEBAllos BARBANCHO - $S$. SCHNABEL

\section{Conclusiones}

La porosidad y densidad medias de la cuenca de Guadalperalón son respectivamente $51,5 \%$ y $1,26 \mathrm{gr} \mathrm{cm}^{3}$, apreciándose una distribución espacial de las mismas similar a la establecida para el estudio de la vegetación. Mientras los suelos más densos y menos porosos se corresponden con las unidades que presentan un mayor nivel de degradación, los menos densos y más porosos se sitúan en áreas con presencia continuada de pastos y de materia orgánica en la superficie.

La evolución temporal de las precipitaciones diarias y la humedad del suelo ha permitido delimitar un umbral de máxima humedad del suelo en torno al 25 \%. La distribución espacial de la humedad en la cuenca no es uniforme y coincide con la división de unidades establecida para el estudio de la vegetación y porosidad-densidad.

El nivel de correlación entre porosidad y humedad es alto y los malos coeficientes $\mathbf{r}^{2}$ se deben a comportamientos muy concretos: existencia de costras superficiales que dificultan la infiltración y permanencia temporal de valores de humedad más o menos constantes en la zona de acumulación de la cuenca.

El ganado influye en el encostramiento de algunas áreas muy localizadas (veredas utilizadas por las ovejas en sus desplazamientos) con un incremento apreciable de la densidad del suelo.

\section{Agradecimientos}

Este estudio ha sido financiado por la Comisión Interministerial de Ciencia y Tecnología, en el marco del proyecto AMB95/0986-CO2-02, y apoyado por la Junta de Extremadura, Dirección General de Enseñanzas Universitarias e Investigación. Agradecer a los Drs A. Cerdà y F. Gallart su atención a las consultas efectuadas. 


\section{Referencias bibliográficas}

Ceballos BARBAncho, A., 1997. Balance de agua de una cuenca bidrográfíca bajo explotación de debesa en Extremadura. Tesis Doctoral. Dp to. Geografia y O.T. Universidad de Extremadura. Cáceres.

CERDÀ, A., 1995. Factores y variaciones espacio-temporales de la infiltración en los ecosistemas mediterráneos. Editorial Geoforma, Logroño.

CERDÀ, A., 1996. Régimen hídrico en los suelos en zonas acarcavadas. Algunos ejemplos en la cuenca experimental de Petrer. Alicante. En GRANDAL, A. y PAGÉS, J. (Eds): IV Reunión de Geomorfología, S.E.G. Cadernos del Laboratorio Xeolóxico de Laxe, 21, 685-694, A Coruña.

Courtney, F.M. y Trudgill, S.T., 1984. The soil. An introduction to soil study. Edward Arnold, London.

DRIESSEN, P.M. Y DUDAL, R., 1991. The major soils of the world. Lecture Notes on their Geography, Formation, Properties and Use. Agricultural University Wageningen y Katholieke Universiteit Leuven, Netherlands.

Duchaufour, P., 1987. Manual de Edafología. Masson, Barcelona.

Dunin, F.X. Y AsTON, A.R., 1981. Spatial variability in the water balance of an experimental catchment. Aust. J. Soil Res., 19: 113-120.

Gamougoun, N.D.; SMith, R.P.; WoOd, K. Y PIEPER, R.D., 1984. Soil vegetation, and hydrologic responses to grazing management at Fort Stanton, New Mexico. Journal of Range Management, 37(6): 538-541.

Gómez AMELIA, D., 1985. La penillanura cacereña. Estudio geomorfológico. Dpto de Geografía. Facultad de Filosofía y Letras. Servicio de Publicaciones. Universidad de Extremadura.

Gómez Amelia, D. y Schnabel, S., 1992. Procesos sedimentológicos e hidrológicos en una pequeña cuenca bajo explotación de dehesa en Extremadura. En López Bermúdez, F.; Conesa García, C. y Romero Díaz, M.A. (Eds): II Reunión Nacional de Geomorfología, 55-63. S.E.G., Murcia.

Gómez Amelia, D. y Schnabel, S., 1996. Hidrología y erosión en ambientes de pastoreo extensivo. En LASANTA, T. Y GARCía RuIz, J.M. (Eds): Erosión y recuperación de tierras en áreas marginales, 137-154. Instituto de Estudios Riojanos y Sociedad Española de Geomorfología.

Guitián Ojea, F. x Carballas Fernández, T., 1976. Técnicas de análisis de suelos. Editorial Pico Sacro, Santiago de Compostela.

HARR, R.D., 1977. Water flux in soil and subsoil on a steep forested slope. Journal of Hydrology, 33: 37-58.

IMESON, A.C., 1984. Desertification in Europe. En R. FANTECHI and N.S. MARGARIS (Edrs): Proceedings of the Information Symposium in the EEC Programme on Climatology. Mytilene, Greece. 
A. CEBALLOS BARBANCHO - S. SCHNABEL

IngELmo SÁNCHEZ, F. y CUADRAdo SÁNCHEZ, S., 1986. El agua y el medio físico del suelo. Centro de Edafología y Biología Aplicada (CSIC). Excma Diputación Provincial, Salamanca.

Ladero Álvarez, M., 1987. La España Luso-Extremadurense. En Peinado Lorca, M. y RIVAS-MARTíneZ, S. (Eds): La vegetación de España, 455-488. Colección Aula Abierta, Universidad de Alcalá.

Martínez Fernández, J., 1994. Estimación de las propiedades físicas de los suelos a partir de variables edáficas de medición sencilla. En ARNÁEZ VADILLO, J.; García Ruiz, J.M. y Gómez ViLlar, A. (Eds): III Reunión de Geomorfología, 317-326. S.E.G., Logroño.

Martínez Fernández, J. y López Bermúdez, F., 1996. Métodos para el estudio de las propiedades hídricas de suelos y formaciones superficiales. Cuadernos Técnicos de la S.E.G. Geoforma Ediciones, Logroño.

Mulholland, B. y Fullen, M.A., 1991. Cattle trampling and soil compactation on loamy sands. Soil use and management, 7(4): 189-193.

Navas, A.M., 1988. La participación de los yesos en la salinización de las aguas superficiales en la cuenca del Ebro. Tesis Doctoral. Universidad de Zaragoza. $466 \mathrm{pp}$.

Price, A.G. Y BaUer, B.O., 1984. Small-scale heterogeneity and soil-moisture variability in the unsaturated zone. Journal of Hydrology, 70: 277-293.

ReYnolds, S.G., 1970. The gravimetric method of soil moisture determination. Part I. A study of equipment and methodological problems. Journal of Hydrology, 11: 258-273.

Romero Díaz, M. A., 1986. Variaciones espaciales de infiltración y su relación con la textura de los suelos en el NE de la provincia de Granada. En: Estudios sobre Geomorfología del Sur de España. 121-126. Murcia.

SCHNABEL, S., 1995. Hydrologiscbe und erosive Prozesse in einem kleinen Einzugsgebiet unter silvo-pastoraler Landnutzung in Extremadura, Spanien. Tesis Doctoral. Freie Universität Berlin.

SCHNABEL, S., 1997. Soil erosion and runoff production in a small watershed under silvo-pastoral landuse (debesas) in Extremadura, Spain. Geoforma Ediciones, Logroño.

ZhANG, T. y Berndtsson, R., 1988. Temporal patterns and spatial scale of soil water variability in a small humid catchment. Journal of Hydrology, 104: 111-128. 